\title{
MODELO DE KURAMOTO E A VERIFICAÇÃO DA DIFERENÇA DE FASE USANDO UMA METODOLOGIA BASEADA NA TRANSFORMADA WAVELET COMPLEXA DUAL-TREE: RESULTADOS PRELIMINARES
}

\author{
Maria Teodora Ferreira*, Celso B. N. Freitas*, Margarete O. Domingues ${ }^{\dagger}$, Elbert E. N. \\ $\mathrm{MACAU}^{\dagger}$ \\ *Programa de Pós-Gradução em Computação Aplicada - CAP/INPE \\ São José dos Campos, SP, Brasil \\ ${ }^{\dagger}$ Laboratório Associado de Matemática Aplicada e Computação - LAC/INPE \\ São José dos Campos, SP, Brasil
}

Emails: mteodoraf25@gmail.com, cbnfreitas@gmail.com, margarete@lac.inpe.br, elbert@lac.inpe.br

\begin{abstract}
The calculation of the phase difference between non-identical systems from the time series is a great challenge. In this article, we explore a method based on Dual-Tree Complex Wavelet Transform in order to calculate the phase difference between three oscillators that follow the Kuramoto model, which has been described in phase variables, allowing to directly assess the accuracy of the method studied.
\end{abstract}

Keywords - Phase Difference, Time Series Analysis, Wavelet Transform.

Resumo- O cálculo da diferença de fase entre sistemas não idênticos a partir da série temporal é um grande desafio. Neste artigo, vamos explorar um método baseado na Transformada Wavelet Complexa Dual-Tree, a fim de calcular a diferença de fase entre três osciladores que seguem o modelo de Kuramoto, o qual já descrito em variáveis de fase, o que nos permite avaliar diretamente a precisão do método estudado.

Palavras-chave— Diferença de Fase, Análise de Séries Temporais, Transformada Wavelet.

\section{Introdução}

Entende-se fase como uma quantidade que cresce $2 \pi$ dentro de um ciclo oscilatório, proporcional a fração do período. A fase determina o estado de um oscilador periódico, parametrizando a forma da onda dentro do ciclo. A fase não fornece nenhuma informação nova sobre o sistema, mas sua vantagem se torna evidente se levarmos em conta a diferença de fase entre sistemas. Isso ajuda a distinguir entre diferentes regimes de sincronização (Pikovsky et al., 2003).

Com o objetivo de calcular a fase de sistemas, pode-se utilizar medidas diretas do ângulo de fase em uma projeção do atrator, bem como técnicas mais sofisticadas tais como: transformada de Hilbert, superfície de seção de Poincaré (Pikovsky et al., 1996), curvatura e plots de recorrência (Romano et al., 2005; Kurths et al., 2006), conjuntos localizados (Pereira et al., 2007), transformada de Fourier de tempo curto e a transformada wavelet contínua usando a wavelet complexa de Morlet (Hramov and Koronovskii, 2004; Koronovskii and Hramov, 2004; Postnikov, 2009). No entanto, nenhuma dessas técnicas trabalha bem em todas as situações.

Em (Ferreira et al., 2011; Ferreira et al., 2013) propõe-se uma abordagem para extrair a fase de sistemas caóticos baseada na Transformada Wavelet Complexa Dual-Tree (DT-CWT), criada por Nick Kingsbury (Selesnick et al., 2005).

Neste trabalho explora-se o método desenvolvido utilizando a DT-CWT para atribuição da di- ferença de fase em sistemas oscilatórios. O objetivo deste trabalho é ilustrar a aplicabilidade e a precisão desta técnica, comparando os resultados com o método tradicional baseado na função arctan.

Na próxima seção apresenta-se a metodologia utilizada para a verificação da diferença de fase. Em seguida, apresentamos os resultados e a análise dos experimentos numéricos. E, por fim, as conclusões deste trabalho.

\section{Metodologia}

Nesta seção é apresentada a formulação do Modelo de Kuramoto (MK), do método baseado na função arctan e do método baseado na DT-CWT.

\subsection{Modelo de Kuramoto}

Em nossa formulação do Modelo de Kuramoto (MK) considera-se que cada oscilador $i=1, \ldots, N$ possui dinâmica determinada pela seguinte equação diferencial

$$
\dot{\phi}_{i}=\omega_{i}-k \sum_{j=1}^{N} A_{i j} \sin \left(\phi_{i}-\phi_{j}\right),
$$

onde $\phi_{i}(t)$ é a variável de fase do $i$-ésimo oscilador, assumindo valores na reta real $\mathbb{R}$, mas podendo também ser visto como um ângulo na circunferência unitária, tomando-se $\phi_{i}(t) \bmod 2 \pi$. As frequências naturais dos osciladores, expressando fisicamente com que velocidade eles evo- 


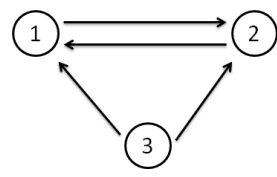

Figura 1: Representação esquemática da topologia de conexão entre os osciladores.

luem quando isolados, são dadas pelos parâmetros $\omega=\left(\omega_{1}, \ldots, \omega_{n}\right) \in \mathbb{R}^{N}$, O grafo direcionado de acoplamento é expresso no sistema pela sua $m a-$ triz de adjacências $A_{N \times N} \doteq\left(A_{i j}\right)$, com $A_{i i}=0$; $A_{i j}=1$, se o oscilador $i$ é influenciado pelo oscilador $j$; ou $A_{i j}=0$, caso contrário. Por último, a constante $k \in \mathbb{R}$ é chamada de força de acoplamento global, ou simplesmente força de acoplamento. Ela ajusta a intensidade da influência exercida por todos os nós conectados diretamente no grafo de acoplamento.

Para as simulações numéricas foi fixado um MK com $N=3$ osciladores, pois desejamos ilustrar como o método baseado na DT-CWT é capaz de detectar em um mesmo experimento dois tipos de sincronização de fase (Pikovsky et al., 2003), a saber: sincronização de fase fixa, quando a diferença de fase entre os osciladores converge para uma constante; e deslizamentos de fase, quando há longos períodos de sincronização de fase fixa intermeados por mudanças bruscas na diferença de fase.

Foi selecionado um grafo de acoplamento com os osciladores 1, 2 acoplados mutuamente e o oscilador 3 agindo como uma forçante para os osciladores 1,2, conforme Figura 1. As frequências naturais e condições inicias são $\omega=(1.00,1.05,1.50)$ e $\phi^{0}=(0,2 \pi / 3,4 \pi / 3)$, respectivamente. Com isso, escolheu-se empiricamente uma força de acoplamento $k=0.475$, de forma que os osciladores 1,2 apresentem sincronização de fase fixa, enquanto os osciladores 1,3 e 2,3 exibem deslizamentos de fase.

É usado o método Preditor-Corretor de Adams-Bashforth-Moulton (Burden and Faires, 2005) para a integração da equação diferencial, utilizando passo de integração de $h=0.01$. O intervalo final de integração é $t_{f}=2 . h .2^{18}$. Contudo, a primeira metade desse intervalo é desconsidera na análise, a fim de eliminar-se um tempo de transiente para o sistema. Por simplicidade, apresentamos a variável de tempo $t$ iniciando em zero e todas as variáveis de fase começando na circunferência unitária.

\subsection{Método baseado na função arctan}

O método usual na literatura para calcular a fase é baseado na projeção do atrator sobre o plano $(x, y)$. A fase $\phi(t)$ é então introduzida como o ângulo no sistema em coordenadas polares sobre o plano $(x, y)$, conforme proposto por (Rosenblum

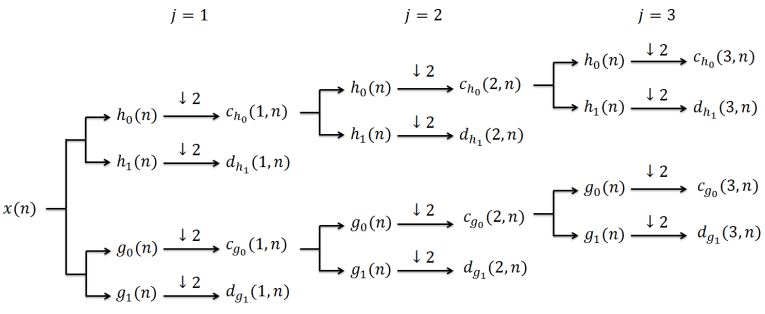

Figura 2: Representação esquemática da decomposição do sinal real $x(n)$ em três escalas $(j=$ $1,2,3)$, utilizando a DT-CWT.

et al., 1996), via a equação:

$$
\phi(t)=\arctan \left(\frac{y}{x}\right) .
$$

Note que para utilizar este método é necessário o conhecimento de duas coordenadas do sistema em estudo; a saber, $x$ e $y$, o que na prática, é inviável, visto que na maioria dos casos tem-se apenas uma coordenada, a saber, a série temporal associada ao sistema.

\subsection{Método baseado na DT-CWT}

A DT-CWT utiliza duas Transformadas Wavelets Discretas (DWT). A DWT é uma transformada real. A primeira DWT é composta por filtros passa-baixa $h_{0}(n)$ e passa-alta $h_{1}(n)$, formando um banco de filtro superior ou árvore superior, conforme observado na Figura 2. Já a segunda DWT é composta de filtros passa-baixa $g_{0}(n)$ e passa-alta $g_{1}(n)$, formando um banco de filtro inferior ou árvore inferior, conforme observado na Figura 2.

Quando considera-se um sinal real $x(n)$, obtém-se $d_{h}(j, n)$ como saída da primeira DWT e $d_{g}(j, n)$ como saída da segunda DWT. Considerando as duas DWT's, obtem-se a wavelet complexa $\psi_{c}(t)=\psi_{h}(t)+\imath \psi_{g}(t)$ e o coeficiente wavelet complexo $d_{c}(j, n)=d_{h}(j, n)+\imath d_{g}(j, n)$.

A Figura 2 apresenta um esquema da decomposição do sinal real $x(n)$ em três escalas $(j=$ $1,2,3)$ utilizando a DT-CWT.

A fim de satisfazer as condições de reconstrução perfeita do sinal, os filtros são construídos de modo que a wavelet complexa seja aproximadamente analítica, ou seja, de modo que $\psi_{g}(t)$ é aproximadamente um par de Hilbert de $\psi_{h}(t)$, denotada como $\psi_{g}(t) \approx \mathcal{H}\left\{\psi_{h}(t)\right\}$, onde $\mathcal{H}$ denota a transformada de Hilbert (Kingsbury, 2000).

Neste trabalho, na primeira escala de decomposição foi utilizado $(13,19)$ tap-filters os quais são biortogonais e quase-simétricos. Para escalas $j>1$ foi utilizados $q$-shift filters com $(14,14)$ tapfilters (Selesnick et al., 2005).

\section{Método para a atribuição da diferença de fase}

A fim de calcular a fase do primeiro sistema, chamado de sistema 1 (veja Figura 1), utilizando 
a abordagem DT-CWT, apenas uma variável de estado, por exemplo $x_{1}$, é analisada. A partir desta análise, obtemos os coeficientes wavelet complexos $d_{c}(j, n)$ em cada escala de decomposição $j$. Depois de obter os coeficientes, a energia $E(j, n)$ em cada escala $j$ é calculada como o módulo ao quadrado dos coeficientes wavelet complexos, ou seja, $E(j, n)=\left|d_{c}(j, n)\right|^{2}$. Após calcular a energia em cada escala, a máxima energia de cada escala $\max _{j} E(j, n)=E(J, n)$ é encontrada com o objetivo de localizar a escala $J$ a qual tem máxima energia. A escala $J$ associada a máxima energia é usada para calcular a fase $\phi(J, n)=\arctan \left(\frac{d_{g}(J, n)}{d_{h}(J, n)}\right)$ em um tempo específico (veja Figura 3). Similarmente, o mesmo procedimento descrito acima é utilizado na série da variável $x$ do sistema 2 e do sistema 3 .

A Figura 3 mostra um esquema do método para calcular a fase de um sistema, utilizando a abordagem da DT-CWT.

Então, a fase de cada sistema é calculada, $\phi_{1}(J, n), \phi_{2}(J, n)$ e $\phi_{3}(J, n)$. Em seguida a diferença de fase entre os sistemas é calculada entre os osciladores 1 e 2 como $\Delta \phi_{12}(t)=\phi_{2}(J, n)-$ $\phi_{1}(J, n)$; entre os osciladores 1 e 3 como $\Delta \phi_{13}(t)=$ $\phi_{3}(J, n)-\phi_{1}(J, n)$ e entre os osciladores 2 e 3 como $\Delta \phi_{23}(t)=\phi_{3}(J, n)-\phi_{2}(J, n)$.

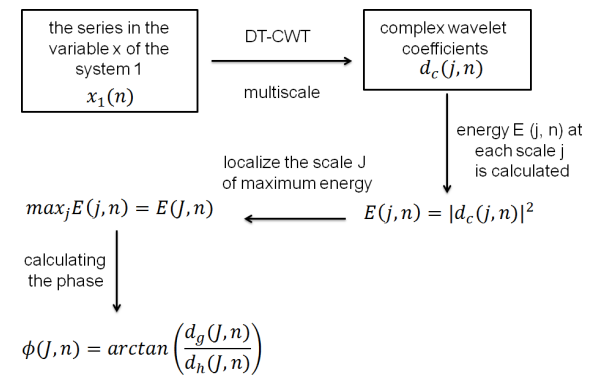

Figura 3: Representação esquemática do método proposto para calcular a fase do sistema 1 utilizando a variável de estado $x$.

\section{Resultados}

Para testar a aplicabilidade do método e verificar a metodologia, a fase foi calculada utilizando o modelo de Kuramoto, o método usual baseado na função arctan e a metodologia wavelet baseada na DT-CWT. A Figura 4 mostra o espectro wavelet global obtido a partir da análise wavelet dos três osciladores. Note que, para os osciladores 1 e 2, a escala de máxima energia é a escala 9 ; já para o oscilador 3, a escala de máxima energia é a escala 8. Devido a escala de máxima energia do oscilador 3 ser diferente da escala de máxima energia dos osciladores 1 e 2, foram testados dois casos para os três osciladores em estudo, a saber, primeiro caso considerando a escala de máxima energia $J=8$ e o segundo caso considerando $J=9$. Em ambos os casos, os resultados da verificação da diferença de fase foram semelhantes. Neste trabalho, apresentamos o resultado considerando a escala $J=9$ para cálculo da diferença de fase entre os osciladores.

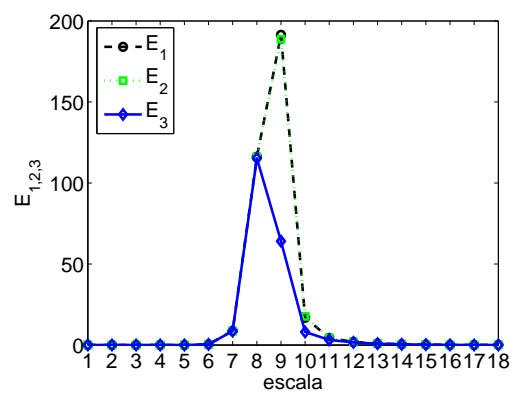

Figura 4: Espectro wavelet global. $E_{i}$ significa a escala de máxima energia associada ao oscilador $i$.

A Figura 5 mostra a diferença de fase entre os osciladores considerando em (a) o modelo de Kuramoto; (b) o método usual e em (c) o método wavelet.

(a)

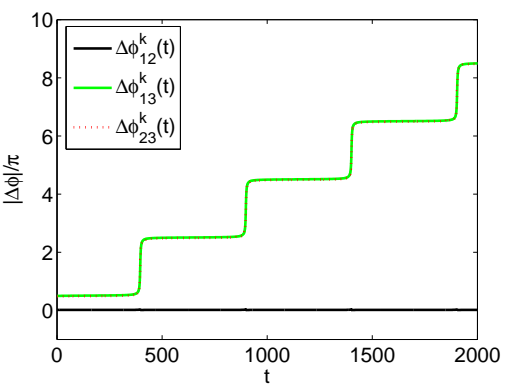

(b)

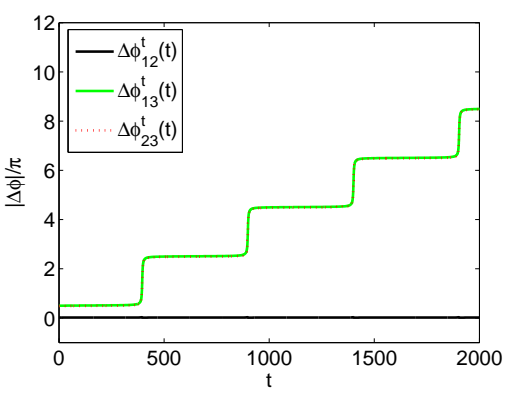

(c)

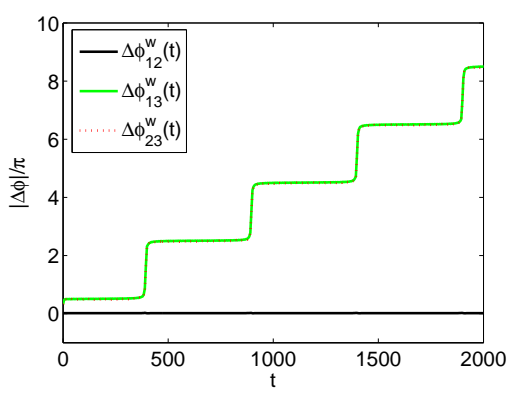

Figura 5: A diferença de fase entre os osciladores considerando em (a) o modelo de Kuramoto; (b) o método usual e em (c) o método wavelet.

A Figura 5 mostra que os osciladores 1,2 es- 
tão sincronizados em fase, já que a diferença de fase $\Delta \phi_{12}(t)$ entre eles é nula. Ademais, o oscilador 3 apresenta deslizamentos de fase em relação aos osciladores 1,2 : a cada intervalo de aproximadamente 500 unidades de tempo, o oscilador 3 dá uma volta adicional sobre os osciladores 1,2, quase como um salto, retornando daí para uma diferença de fase constante de $\pi / 4$. Note que os resultados da diferença de fase entre os osciladores 1 e 3, ou seja, $\Delta \phi_{13}(t)$ e entre os osciladores 2 e 3 , ou seja, $\Delta \phi_{23}(t)$ ficaram sobrepostos na Figura 5 Analisando o método baseado na função arctan e o baseado na DT-CWT, verifica-se que ambos os métodos foram capazes de reconstruir a diferença de fase de referência do MK, permitindo a correta identificação dos regimes de sincronização entre os osciladores.

\section{Conclusões}

Neste artigo foi proposto e executado um teste de verificação para atribuição da diferença de fase utilizando o método wavelet baseado na DTCWT. Ilustramos com um modelo simples que a técnica proposta é bastante confiável, produzindo resultados qualitativamente tão bons ou melhores quanto os resultados do método usual. A principal vantagem na utilização do método wavelet consiste no fato de não ser necessário duas variáveis de estado, por exemplo $x$ e $y$, para o cálculo da fase. Essa vantagem motiva a futuras aplicações do método na análise de séries temporais experimentais.

\section{Agradecimentos}

Este trabalho é apoiado pela CAPES, CNPQ e FAPESP.

\section{Referências}

Burden, R. L. and Faires, J. D. (2005). Numerical Analysis, Available Titles CengageNOW Series, Brooks/Cole.

Ferreira, M. T., Follmann, R., Domingues, M. O. and Macau, E. E. N. (2011). Transformada wavelet complexa dual-tree e a detecção da sincronização de fase entre sistemas com dinâmica caótica, 1: 723-726.

Ferreira, M. T., Follmann, R., Domingues, M. O. and Macau, E. E. N. (2013). Dual-tree complex wavelet transform as an automatic tool to detect phase synchronization between systems with chaotic dynamics. Artigo não publicado.

Hramov, A. and Koronovskii, A. (2004). An approach to chaotic synchronization, Chaos 14(3): 603-610. DOI: 10.1063/1.1775991
Kingsbury, N. (2000). A dual-tree complex wavelet transform with improved orthogonality and symmetry properties.

Koronovskii, A. and Hramov, A. (2004). Chaotic phase synchronization studied by means of continuous wavelet transform, Technical Phy-sics Letters 30: 587-590. DOI: $10.1134 / 1.1783411$

Kurths, J., Romano, M., Thiel, M., Osipov, G., Ivanchenko, M., Kiss, I. and Hudson, J. (2006). Synchronization analysis of coupled noncoherent oscillators, Nonlinear Dynamics 44: 135-149. DOI: 10.1007/s11071-006-1957-x

Pereira, T., Baptista, M. and Kurths, J. (2007). General framework for phase synchronization through localized sets, Phys. Rev. E 75(2): 026216. DOI: 10.1103/PhysRevE.75.026216

Pikovsky, A., Rosenblum, M. and Kurths, J. (1996). Synchronization in a population of globally coupled chaotic oscillators, Europhysics Letters 34(3): 165-170. DOI: 10.1209/epl/i1996-00433-3

Pikovsky, A., Rosenblum, M. and Kurths, J. (2003). Synchronization: A Universal Con-cept in Nonlinear Sciences, Cambridge Uni-versity Press, New York.

Postnikov, E. (2009). Wavelet phase synchronization and chaoticity, Phys. Rev. E 80: 057201. DOI: 10.1103/PhysRevE.80.057201

Romano, M., Thiel, M., Kurths, J., Kiss, I. and Hudson, J. (2005). Detection of synchronization for non-phase-coherent and nonstationary data, EPL (Europhysics Letters) 71(3): 466. DOI: 10.1209/epl/i2005-10095-1

Rosenblum, M., Pikovsky, A. and Kurths, J. (1996). Phase synchronization of chaotic oscillators, Phys. Rev. Lett. 76(11): 1804-1807. DOI: 10.1103/PhysRevLett.76.1804

Selesnick, I., Baraniuk, R. and Kingsbury, N. (2005). The dual-tree complex wavelet transform, IEEE Signal Processing Magazine 22(6): 123-151. DOI: 10.1109/MSP.2005.1550194 